\title{
Влияние потерь на вывод излучения на динамические характеристики вертикально-излучающих лазеров спектрального диапазона 1.55 мкм, изготовленных методом спекания эпитаксиальных пластин
}

\author{
(C) С.А. Блохин ${ }^{1}$, М.А. Бобров ${ }^{1}$, А.А. Блохин ${ }^{1,2}$, А.Г. Кузьменков ${ }^{2}$, Н.А. Малеев ${ }^{1}$, В.М. Устинов ${ }^{2}$, \\ E.C. Колодезный ${ }^{3}$, C.C. Рочас ${ }^{3}$, А.В. Бабичев ${ }^{3}$, И.И. Новиков ${ }^{3}$, А.Г. Гладышев ${ }^{3}$, \\ Л.Я. Карачинский ${ }^{1,4}$, Д.В. Денисов ${ }^{4,5}$, К.О. Воропаев ${ }^{6,7}$, А.С. Ионов ${ }^{7}$, А.Ю. Егоров ${ }^{3}$ \\ ${ }^{1}$ Физико-технический институт им. А.Ф. Иоффре Российской академии наук, \\ 194021 Санкт-Петербург, Россия \\ ${ }^{2}$ Научно-технологический центр микроэлектроники и субмикронных гетероструктур \\ Российской академии наук, \\ 194021 Санкт-Петербург, Россия \\ ${ }^{3}$ Санкт-Петербургский национальный исследовательский университет \\ информационных технологий, механики и оптики, \\ 197101 Санкт-Петербург, Россия \\ ${ }^{4} \mathrm{OOO}$ „Коннектор Оптикс", \\ 194292 Санкт-Петербург, Россия \\ ${ }^{5}$ Санкт-Петербургский государственный электротехнический университет „ЛЭТИ“ им. В.И. Ульянова (Ленина), \\ 197376 Санкт-Петербург, Россия \\ ${ }^{6}$ Новгородский государственный университет им. Ярослава Мудрого, \\ 173003 Великий Новгород, Россия \\ ${ }^{7}$ ОАО „ОКБ-Планета“, \\ 173004 Великий Новгород, Россия \\ E-mail: blokh@mail.ioffe.ru
}

Поступила в Редакцию 25 марта 2019 г.

В окончательной редакции 1 апреля 2019 г.

Принята к публикации 1 апреля 2019 г.

\begin{abstract}
Представлены результаты исследования динамических характеристик одномодовых вертикально-излучающих лазеров спектрального диапазона 1.55 мкм, полученных методом спекания пластин высокодобротных брэгговских отражателей и активной области на основе тонких сильнонапряженных InGaAs/InAlGaAs квантовых ям. Обнаружено, что предложенные конструкции активной области и оптического микрорезонатора лазера принципиально обеспечивают возможность достижения высокого уровня дифференциального усиления лазера в диапазоне температур $20-85^{\circ} \mathrm{C}$, однако слабая локализация электронов ведет к росту компрессии усиления при повышенных температурах. В результате при температуре $20^{\circ} \mathrm{C}$ частота эффективной модуляции вертикально-излучающих лазеров за счет повышения потерь на вывод излучения может быть увеличена с 9.2 до 11.5 ГГц, тогда как при температуре $85^{\circ} \mathrm{C}$ частота эффективной модуляции не превышает 8.5 ГГц, слабо зависит от потерь на вывод излучения и в основном лимитирована насыщением оптического усиления.
\end{abstract}

Ключевые слова: вертикально-излучающий лазер, спекание пластин, частота модуляции, время жизни фотонов в резонаторе.

DOI: $10.21883 /$ FTP.2019.08.48006.9112

\section{1. Введение}

Длинноволновые вертикально-излучающие лазеры (ВИЛ) спектрального диапазона 1.3/1.55 мкм являются привлекательным вариантом для реализации гибридной интеграции источников лазерного излучения с интегральными фотонными устройствами на основе $\mathrm{Si}$, открывающей перспективы увеличения протяженности скоростных оптических каналов передачи данных и создания различных устройств радиофотоники. К сожалению, классическая конструкция ВИЛ с инжекцией носителей через легированные распределенные брэгговские отражатели (РБО, англ. Distributed Bragg reflector DBR), хорошо зарекомендовавшая себя при создании ВИЛ ближнего ИК-диапазона в системе материалов InAlGaAs/GaAs [1], оказывается непригодна для создания длинноволновых ВИЛ в системе материалов InAlGaAs/GaAs ввиду отсутствия эффективной активной области. Применение выращенной в едином эпитаксиальном процессе монолитной структуры ВИЛ с инжекцией носителей через внутрирезонаторные контактные слои и туннельный переход в системе материалов InAlGaAsP/InP позволяет реализовать длинноволновые ВИЛ, но не обеспечивает хорошей температурной ста- 
бильности характеристик, а эффективная частота модуляции ограничена на уровне $4-5$ ГГц, что обусловлено малым контрастом показателей преломления и низкой теплопроводностью слоев тройных и четверных твердых растворов в РБО, решеточно-согласованных с подложкой $\operatorname{InP}[2]$.

Решение данной проблемы возможно за счет применения гибридной конструкции ВИЛ с инжекцией носителей через внутрирезонаторные контактные слои (англ. intra-cavity contacted layer - IC-layer) и захороненный туннельный переход (англ. buried tunnel junction - BТJ) в сочетании с зеркалами на основе других систем материалов, имеющими высокую добротность и теплопроводность. Один из подходов связан с использованием комбинированного „глухого“ зеркала на основе высококонтрастного диэлектрического $\mathrm{CaF}_{2} / \mathrm{ZnS}$ РБО и толстого слоя золота, выполняющего также функцию теплоотвода, что позволяет реализовать ВИЛ на основе напряженных InAlGaAs квантовых ям (КЯ) спектрального диапазона 1.55 мкм с частотой эффективной модуляции 12 ГГц и скоростью передачи данных 25 Гбит/с при температуре $25^{\circ} \mathrm{C}$ [3]. Уменьшение длины микрорезонатора (за счет применения конструкции с двумя внутрирезонаторными контактными слоями и диэлектрическими РБО) позволило повысить частоту эффективной модуляции ВИЛ до 21 ГГц и продемонстрировать безошибочную передачи данных на скорости 50 Гбит/с при температуре $20^{\circ} \mathrm{C}$ [4], однако такая технология изготовления сложна и требует применения высококачественных диэлектрических зеркал.

Альтернативным подходом является использование технологии спекания пластин гетероструктуры активной области на основе нескольких $\operatorname{In}(\mathrm{Al}) \mathrm{GaAs}$ квантовых ям, выращенной на подожке InP, и двух гетероструктур AlGaAs/GaAs РБО, выращенных на подожках GaAs, которая позволяет объединить преимущества обеих систем материалов [5]. Изготовленные по такой технологии ВИЛ спектрального диапазона 1.5 мкм демонстрируют частоту эффективной модуляции 7-9ГГц и безошибочную передачу данных на скорости 10 Гбит/с при температуре $20^{\circ} \mathrm{C}$ [6]. Сравнительно недавно с использованием метода спекания пластин нами была продемонстрирована возможность создания эффективных ВИЛ спектрального диапазона 1.55 мкм на основе тонких сильнонапряженных InGaAs/InAlGaAs КЯ, пригодных для безошибочной передачи данных на скорости не менее 25 Гбит/с при температуре $20^{\circ} \mathrm{C}[7,8]$. При этом в ряде работ была показана важность контроля времени жизни фотонов в резонаторе для снижения эффекта демпфирования релаксационных колебаний и повышения быстродействия ВИЛ ближнего ИК-диапазона $[9,10]$.

В настоящей работе представлены результаты анализа влияния времени жизни фотонов в оптическом микрорезонаторе на динамические характеристики одномодовых ВИЛ спектрального диапазона 1.55 мкм, полученных методом спекания двух пластин $\mathrm{AlGaAs} / \mathrm{GaAs}$ РБО и пластины с активной областью на основе тонких сильнонапряженных InGaAs/InAlGaAs КЯ.

\section{2. Детали эксперимента}

Конструкция исследуемых ВИЛ спектрального диапазона 1.55 мкм представляет собой вертикальный микрорезонатор с выводом излучения вверх, который содержит два внутрирезонаторных контакта, а также захороненный туннельный переход (ЗТП) для обеспечения токового и оптического ограничения. Гетероструктура ВИЛ сформирована с помощью технологии спекания пластин, выращенных методом молекулярнопучковой эпитаксии (МПЭ) на подложке InP (активная область) или GaAs (верхний или нижний $\mathrm{AlGaAs} / \mathrm{GaAs}$ РБО). Детальное описание эпитаксиальных гетероструктур активной области и РБО приведено в работе [11]. В активной области использованы тонкие сильнонапряженные $\mathrm{In}_{0.74} \mathrm{Ga}_{0.26}$ As КЯ (параметр рассогласования кристаллической решетки $\sim 1.4 \%$ ), разделенные решеточно-согласованными барьерными слоями $\mathrm{In}_{0.53} \mathrm{Al}_{0.20} \mathrm{Ga}_{0.27} \mathrm{As}$ (соответствующая величина продольного фактора оптического ограничения 0.011). Такая активная область позволяет обеспечить эффективную локализацию электронов на основном уровне КЯ (оценочные значения энергетического разрыва в зоне проводимости и энергии локализации 0.25 и 0.1 эВ соответственно) и уменьшить плотность состояний в валентной зоне за счет расщепления энергетических подзон дырок, вызванного механическим напряжением слоев.

Время жизни фотонов в резонаторе зависит от внутренних оптических потерь $A_{\text {int }}$ и потерь на вывод излучения $T_{m}$ следующим образом [12]:

$$
\tau_{p}^{-1}=\left(A_{\text {int }}+T_{m}\right) v_{g r},
$$

где $v_{g r}-$ групповая скорость фотонов $\left(\sim 1 \cdot 10^{10} \mathrm{~cm} / \mathrm{c}\right)$. Поскольку потери на вывод излучения определяются коэффициентами отражения зеркал $R$ и длиной резонатоpa $L_{c}$ как $T_{m}=-0.5 \ln \left(R_{t} \cdot R_{b}\right) / L_{c}$, модификация времени жизни фотонов в резонаторе возможна путем изменения коэффициента отражения верхнего РБО за счет осаждения низкодобротного диэлектрического $\mathrm{SiO}_{2} / \mathrm{Ta}_{2} \mathrm{O}_{5}$ РБО (формируется гибридный верхний РБО). При этом необходимо учитывать конечную глубину проникновения электромагнитного поля в РБО. Согласно результатам расчета распределения электромагнитного поля в структуре длинноволнового ВИЛ, эффективная длина вертикального резонатора достигает $\sim 3.4$ мкм. Благодаря низкому уровню легирования внутрирезонаторных контактных слоев уровень внутренних оптических потерь не превышает $6.5 \mathrm{~cm}^{-1}$ при температуре $20^{\circ} \mathrm{C}$ и $9.5 \mathrm{~cm}^{-1}$ при температуре $85^{\circ} \mathrm{C}$ соответственно [11]. На рис. $1, a$ представлены результаты моделирования времени жизни фотонов в резонаторе ВИЛ в зависимости от суммарных потерь на вывод излучения. Видно, что уменьшение 

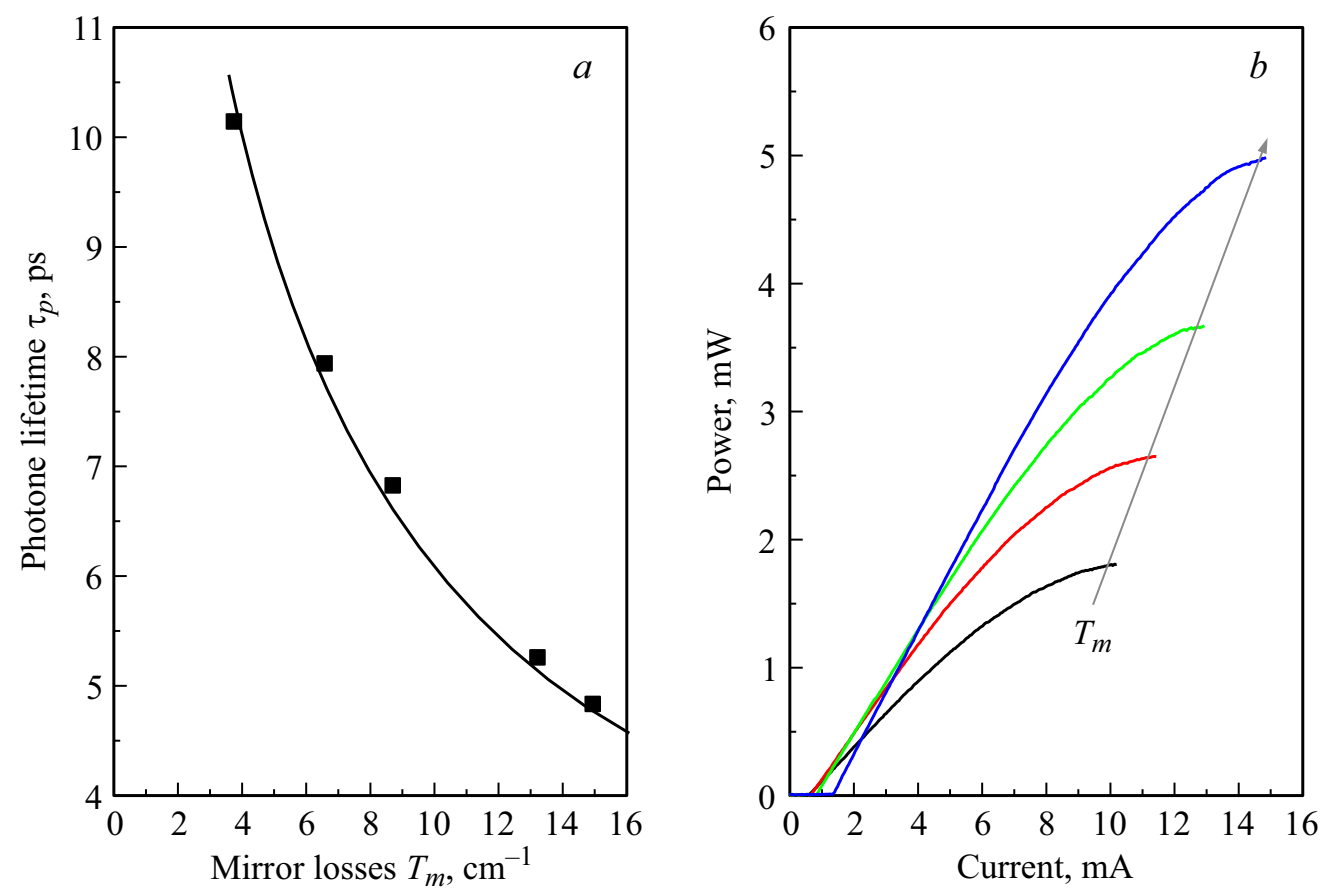

Рис. 1. Расчетная зависимость времени жизни фотонов в резонаторе от уровня потерь на вывод излучения $(a)$ и ватт-амперные характеристики длинноволнового ВИЛ с различным уровнем потерь на вывод излучения, измеренные при температуре $20^{\circ} \mathrm{C}(b)$.

потерь на вывод излучения вследствие роста коэффициента отражения верхнего гибридного РБО ведет к существенному повышению времени жизни фотонов в резонаторе.

\section{3. Результаты и обсуждение}

На рис. $1, b$ приведены вольт- и ватт-амперные характеристики длинноволновых ВИЛ с диаметром ЗТП 8 мкм, измеренные в непрерывном режиме работы при температуре $20^{\circ} \mathrm{C}$. Исходные ВИЛ (т.е. без дополнительного верхнего диэлектрического РБО) обладают высокими потерями на вывод излучения и демонстрируют одномодовую лазерную генерацию с пороговым током $\sim 1.4-1.5$ мА и дифференциальной эффективностью $\sim 0.48$ Вт/А. По мере уменьшения потерь на вывод излучения за счет увеличения коэффициента отражения гибридного РБО наблюдается снижение дифференциальной эффективности до $\sim 0.26 \mathrm{BT} / \mathrm{A} \mathrm{и} \mathrm{падение} \mathrm{порогового}$ тока до 0.6 мА. Следует отметить, что несмотря на большое проектное рассогласование спектра усиления КЯ и резонансной длины волны резонатора ( 50-60 нм), с ростом температуры наблюдается резкое увеличение порогового тока, что, по-видимому, обусловлено термическим выбросом носителей из узкой InGaAs КЯ.

Согласно результатам малосигнального частотного анализа, предельно достижимая частота эффективной модуляции (по уровню спада модуляционной характеристики на -3 дБ) длинноволновых ВИЛ с малыми потерями на вывод излучения при температуре $20^{\circ} \mathrm{C}$ не превышает 9.2ГГц, тогда как по мере роста потерь на вывод излучения наблюдается ее рост до 11.5 ГГц (см. рис. 2,a). Однако повышение температуры измерений до $85^{\circ} \mathrm{C}$ нивелирует положительный эффект от увеличения потерь на вывод излучения, в результате частота эффективной модуляции при повышенных температурах слабо зависит от потерь на вывод излучения и не превышает 8.5 ГГц (см. рис. 3,a).

Для адекватного анализа динамических характеристик ВИЛ на основе данных измерений коэффициента отражения модулирующего СВЧ-сигнала (параметр $S_{11}$ ) в зависимости от частоты была определена величина частоты LRC-фильтра нижних частот, образованного паразитными элементами эквивалентной схемы лазера. Установлено, что во всем диапазоне рабочих токов паразитный LRC-фильтр не является фактором, лимитирующим быстродействие исследуемых ВИЛ.

На рис. $2, b$ и $3, b$ представлены зависимости резонансной частоты $f_{R}$ от тока накачки для двух крайних случаев (лазеры с высокими и низкими потерями на вывод излучения). Уменьшение потерь на вывод излучения ведет к росту скорости нарастания резонансной частоты с ростом тока накачки выше порога генерации (так называемый $D$-фактор), что позволяет достичь более высоких резонансных частот. Согласно [12], D-фактор можно представить как

$$
D=\frac{f_{R}}{\sqrt{I-I_{\mathrm{th}}}}=\frac{1}{2 \pi} \sqrt{\frac{\eta_{j} g_{N} c}{q n_{g} V_{p}}},
$$

где $c$ - скорость света, $q-$ заряд электрона, $\eta_{j}-$ эффективность токовой инжекции, $g_{N}$ - дифференци- 

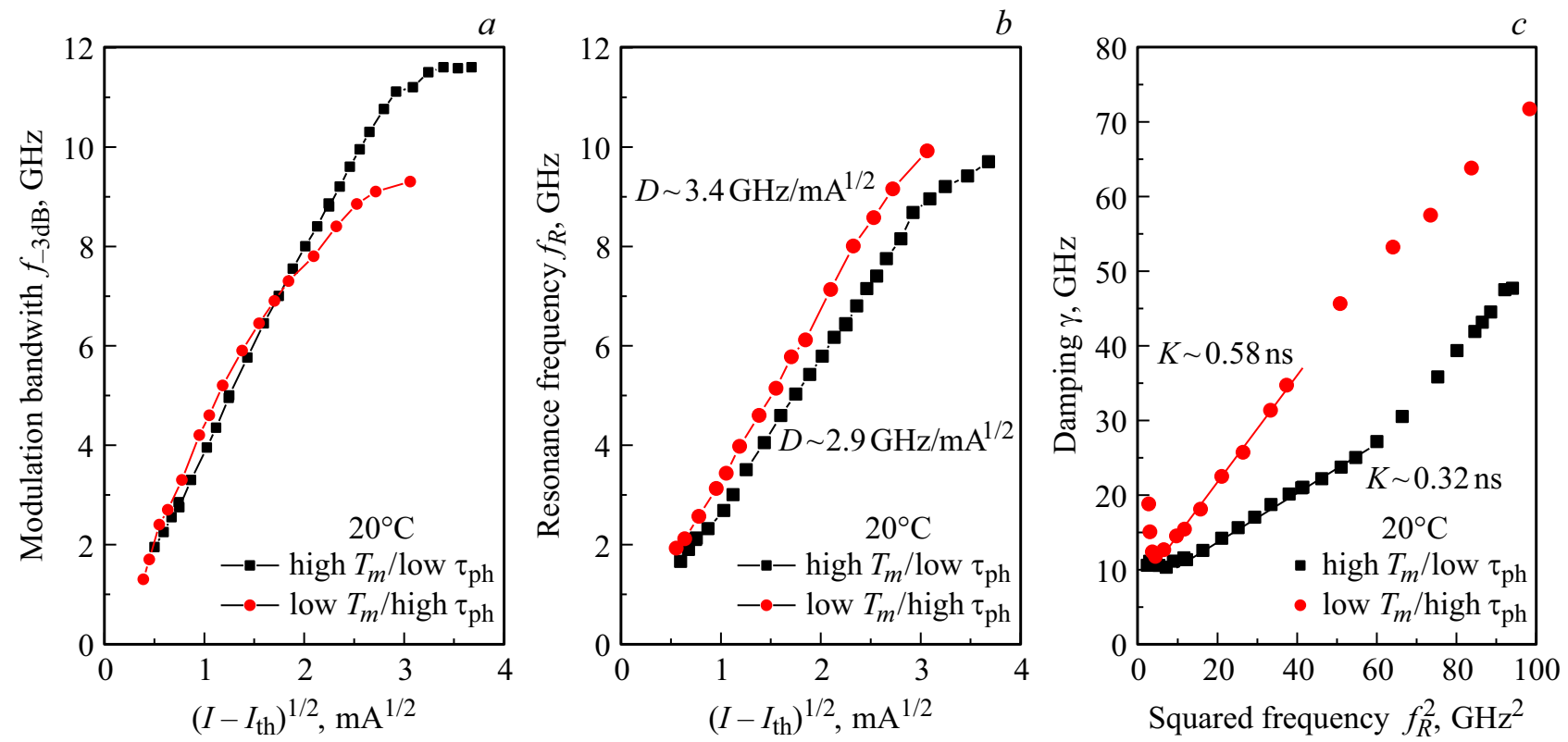

Рис. 2. Результаты малосигнального частотного анализа ВИЛ с различным уровнем потерь на вывод излучения, измеренные при температуре $20^{\circ} \mathrm{C}: a-$ зависимость частоты эффективной модуляции от $\sqrt{I-I_{\mathrm{th}}}, b-$ зависимость резонансной частоты от $\sqrt{I-I_{\mathrm{th}}}, c-$ зависимость коэффициента затухания от квадрата резонансной частоты.
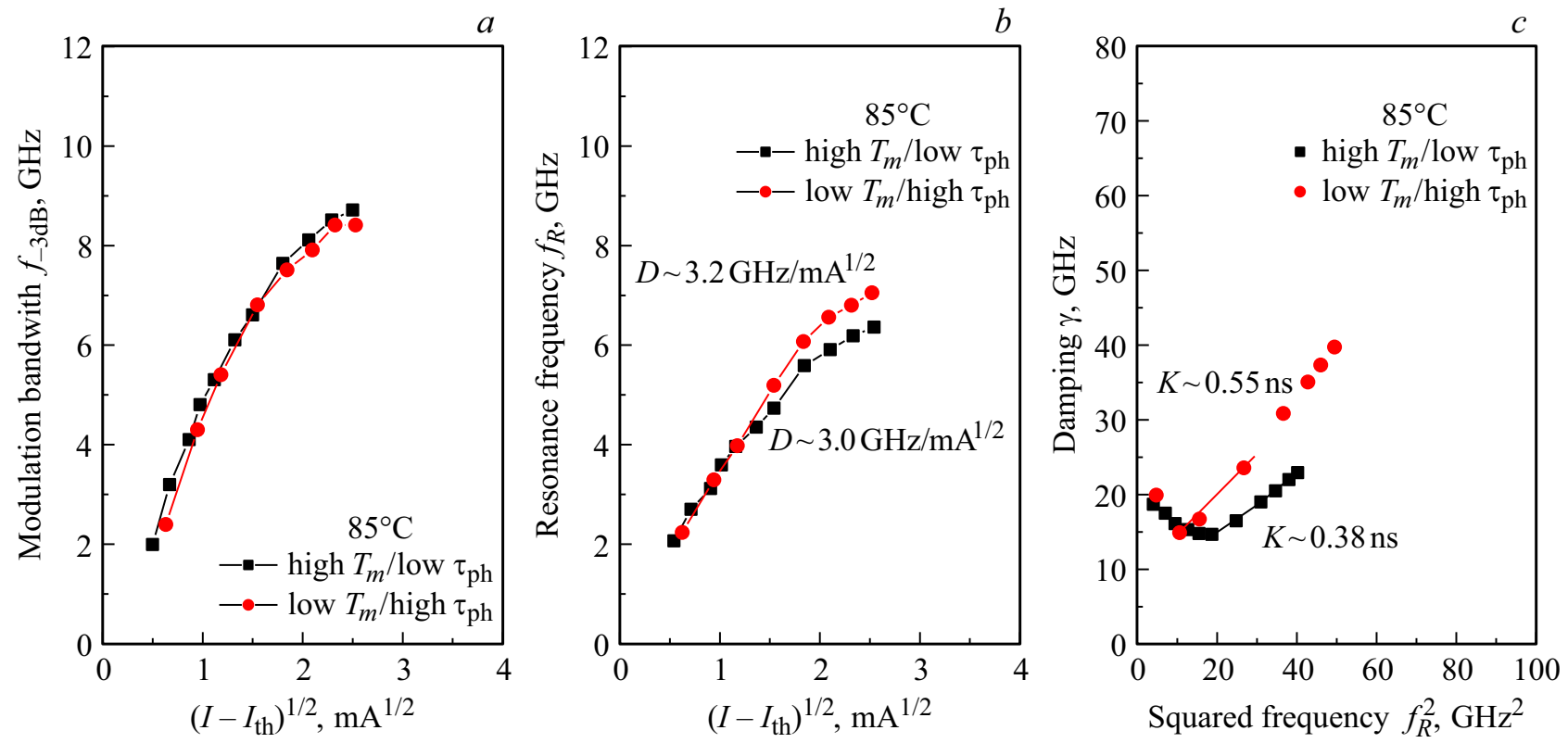

Рис. 3. Результаты малосигнального частотного анализа ВИЛ с различным уровнем потерь на вывод излучения, измеренные при температуре $85^{\circ} \mathrm{C}: a-$ зависимость частоты эффективной модуляции от $\sqrt{I-I_{\mathrm{th}}}, b-$ зависимость резонансной частоты от $\sqrt{I-I_{\text {th }}}, c-$ зависимость коэффициента затухания от квадрата резонансной частоты.

альное усиление на пороге, $n_{g}$ - групповой показатель преломления $(\sim 3), V_{p}-$ объем моды, пропорциональный площади захороненного туннельного перехода и эффективной длине резонатора $\left(\sim 1.6 \cdot 10^{-10} \mathrm{~cm}^{-3}\right)$. Данное соотношение можно использовать для определения зависимости величины дифференциального усиления от тока накачки. При этом для предложенной конструкции ВИЛ изменением эффективности токовой инжекции или объема моды при изменении потерь на вывод излучения можно пренебречь. Данные по эффективности токовой инжекции были взяты из работы [11]. При сравнимом уровне потерь на вывод излучения полученное дифференциальное усиление в 3-4 раза выше значений $g_{N}$ для ВИЛ спектрального диапазона 

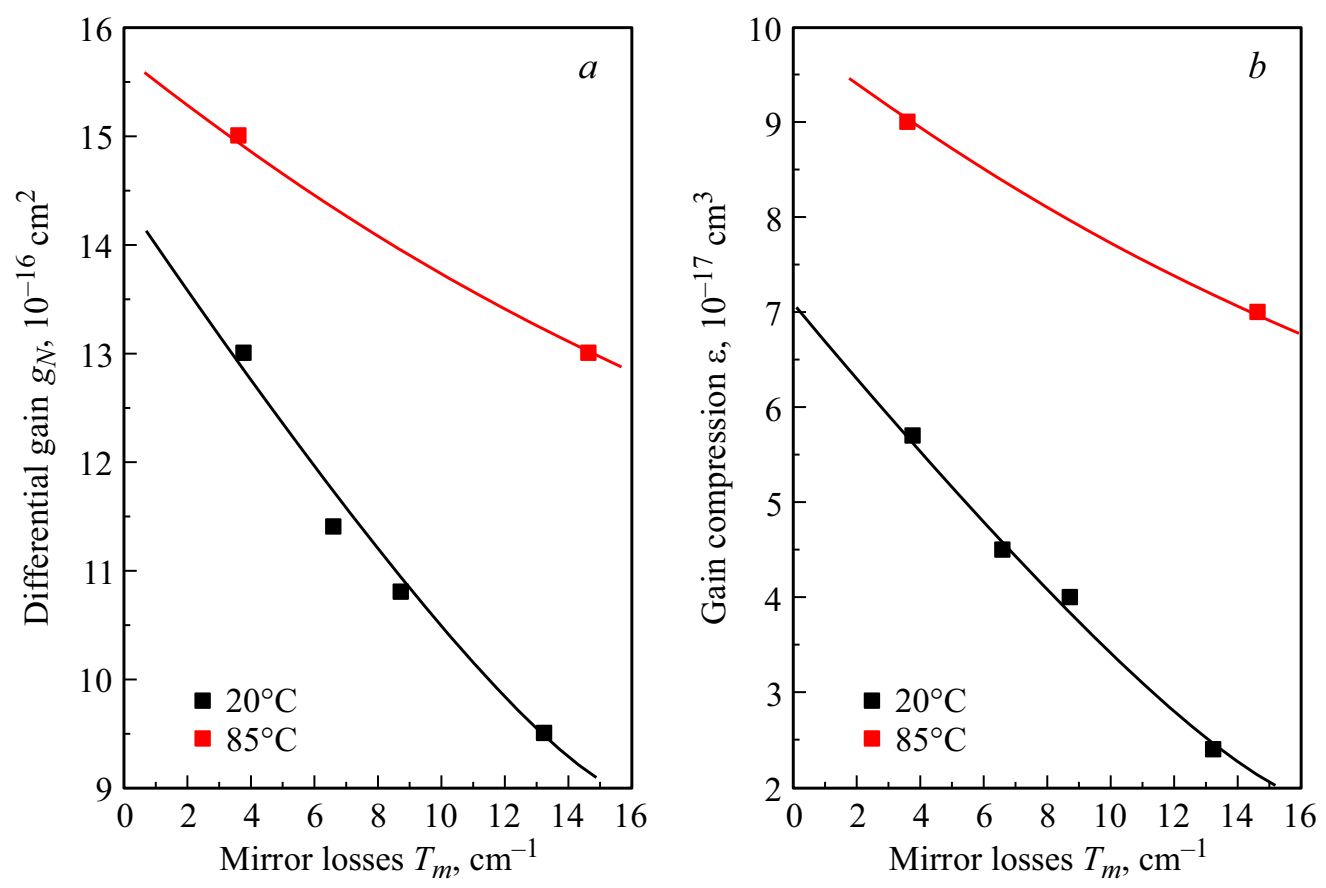

Рис. 4. Зависимости дифференциального усиления $(a)$ и компрессии усиления $(b)$ от уровня потерь на вывод излучения, полученные при температуре 20 и $85^{\circ} \mathrm{C}$.

1.3 мкм на основе InAlGaAs/InAlGaAs КЯ с упругими механическими напряжениями $\sim 1 \%[13]$ и сравнимо со значением $g_{N}$ для ВИЛ спектрального диапазона 1.55 мкм на основе InAlGaAs/InAlGaAs КЯ с упругими механическими напряжениями $\sim 1.2 \%$ [14]. Рост потерь на вывод излучения ведет к падению дифференциального усиления (см. рис. 4,a) и, как следствие, к заметному снижению значения $D$-фактора. При увеличении температуры, несмотря на существенный рост порогового тока, дифференциальное усиление возрастает и слабее зависит от уровня потерь на вывод излучения благодаря проектному рассогласованию спектра усиления активной области и резонансной длины волны. Дело в том, что более высокое дифференциальное усиление достигается на коротковолновой части спектра усиления активной области [15]. Поскольку по мере увеличения температуры рассогласование спектра усиления активной области и резонансной длины волны уменьшается, это ведет к возможности получения более высокого дифференциального усиления при повышенной температуре. Однако падение эффективности токовой инжекции и повышение уровня внутренних оптических потерь [11], а также рост теплового сопротивления при увеличении температуры ведут к более быстрому тепловому загибу зависимости резонансной частоты от тока накачки, и эффект влияния изменения потерь на вывод излучения на величину дифференциального усиления становится слабо выраженным.

На рис. 2, с и 3, с представлены зависимости коэффициента затухания релаксационных колебаний от квадрата резонансной частоты для ВИЛ с высокими и низкими потерями на вывод излучения. Обнаруженная нелинейность при малых плотностях фотонов обусловлена вкладом спонтанного излучения в лазерную моду при токах накачки вблизи порога генерации, а нелинейность при более высоких плотностях фотонов саморазогревом лазера и насыщением усиления. При сравнимой плотности фотонов (т. е. близкой резонансной частоте) лазеры с высокими потерями на вывод излучения обладают меньшим коэффициентом затухания релаксационных колебаний благодаря меньшему времени жизни фотонов в резонаторе. Следует отметить, что повышение температуры слабо влияет на характер данных зависимостей.

Для оценки коэффициента компрессии усиления можно воспользоваться выражением для $K$-фактора [12]:

$$
K=\frac{\gamma-\gamma_{0}}{f_{R}^{2}}=4 \pi^{2}\left(\tau_{p}+\frac{\varepsilon n_{g} \chi}{g_{N} c}\right),
$$

где $\varepsilon-$ коэффициент нелинейности усиления активной области, $\chi-$ транспортный коэффициент $(\sim 1)$, $\gamma_{0}$ - смещение коэффициента затухания. На рис. $4, b$ приведены расчетные значения коэффициента компрессии усиления с учетом изменения дифференциального усиления и времени жизни фотонов в резонаторе при вариации потерь на вывод излучения. Видно, что уменьшение потерь на вывод излучения ведет к существенному росту коэффициента компрессии усиления, что, по-видимому, связано с усилением эффектов разогрева носителей при малой плотности носителей заряда [16]. Для толстых InGaAs/InGaAsP КЯ была показана возможность уменьшения компрессии усиления при улучшении 
локализации носителей за счет использования более высоких барьеров InAlGaAs [17,18]. Однако, несмотря на большой разрыв зон проводимости в используемой узкой InGaAs/InAlGaAs КЯ, найденные коэффициенты компрессии усиления в 2 раза выше значений $\varepsilon$ для ВИЛ спектрального диапазона 1.55 мкм на основе InAlGaAs/InAlGaAs КЯ с упругими механическими напряжениями $\sim 1.2 \%$ при сравнимом уровне потерь на вывод излучения [14]. По-видимому, это обусловлено слабой локализацией электронов в используемой узкой InGaAs/InAlGaAs KЯ, несмотря на большой разрыв зон проводимости. Данное предположение частично подтверждает резкий рост коэффициента компрессии усиления при повышенных температурах, поскольку с ростом температуры не только увеличивается поглощение на свободных носителях, но и усиливается термический выброс носителей из КЯ [19].

\section{4. Заключение}

В работе проведены детальные исследования быстродействия одномодовых ВИЛ спектрального диапазона 1.55 мкм, сформированных методом спекания пластин $\mathrm{AlGaAs} / \mathrm{GaAs}$ РБО и активной области на основе тонких сильнонапряженных InGaAs/InAlGaAs КЯ. Выполненные оценки значения дифференциального усиления и коэффициента компрессии усиления для тонких сильнонапряженных InGaAs/InAlGaAs КЯ позволяют сделать вывод о том, что, несмотря на высокое дифференциальное усиление, слабая локализация электронов ведет к более высокому уровню компрессии усиления по сравнению с толстыми InAlGaAs/InAlGaAs КЯ и, как следствие, к резкому росту компрессии усиления с температурой. В результате при комнатной температуре увеличение потерь на вывод излучения (уменьшение времени жизни фотонов в резонаторе) позволяет повысить частоту эффективной модуляции ВИЛ с 9.2 до 11.5 ГГц, однако при повышенной температуре, несмотря на высокое дифференциальное усиление, рост порогового тока и теплового сопротивления ведут к сильному саморазогреву лазера и быстрому насыщению усиления, что в конечном счете и ограничивает частоту эффективной модуляции ВИЛ на уровне 8.5 ГГц.

Предложенная и реализованная конструкция ВИЛ демонстрирует совокупность параметров, позволяющих надеяться на успешное применение таких ВИЛ в оптоволоконных системах связи, где необходимо обеспечить скорость передачи данных 10-12Гбит/с в диапазоне $20-85^{\circ} \mathrm{C}$. Дальнейшие пути повышения быстродействия для данного типа ВИЛ на основе InGaAs/InAlGaAs КЯ связаны с уменьшением объема моды за счет использования меньших размеров захороненного туннельного перехода и(или) длины эффективного микрорезонатора, а также с минимизацией тепловыделения за счет снижения сопротивления лазера.

\section{Финансирование работы}

Работа выполнена при поддержке Министерства науки и высшего образования России, федеральная целевая программа „Исследования и разработки по приоритетным направлениям развития научно-технологического комплекса России на 2014-2020 годы“, соглашение о предоставлении субсидии от 26.09.2017 г. № 14.578.21.0253, уникальный идентификаTop RFMEFI57817X0253.

\section{Конфликт интересов}

Авторы заявляют, что у них нет конфликта интересов.

\section{Список литературы}

[1] D. Bimberg, A. Larsson, A. Joel. Comp. Semicond., 20 (7), 34 (2014).

[2] M.-R. Park, O.-K. Kwon, W.-S. Han, K.-H. Lee, S.-J. Park, B.-S. Yoo. IEEE Photon. Technol. Lett., 18 (16), 1717 (2006).

[3] W. Hofmann. IEEE Photon. J., 2 (5), 802 (2010).

[4] S. Spiga, W. Soenen, A. Andrejew, D. Schoke, X. Yin, J. Bauwelinck, G. Boehm, M.C. Amann. IEEE J. Lightwave Technol., 35 (4), 727 (2017).

[5] A. Syrbu, A. Mircea, A. Mereuta, A. Caliman, C.-A. Berseth, G. Suruceanu, V. Iakovlev, M. Achtenhagen, A. Rudra, E. Kapon. IEEE Photon. Technol. Lett., 16 (5), 1230 (2004).

[6] D. Ellafi, V. Iakovlev, A. Sirbu, G. Suruceanu, Z. Mickovic, A. Caliman, A. Mereuta, E. Kapon. Opt. Express, 22 (26), 32180 (2014).

[7] A.V. Babichev, L.Ya. Karachinsky, I.I. Novikov, A.G. Gladyshev, S.A. Blokhin, S. Mikhailov, V. Iakovlev, A. Sirbu, G. Stepniak, L. Chorchos, J.P. Turkiewicz, K.O. Voropaev, A.S. Ionov, M. Agustin, N.N. Ledentsov, A.Yu. Egorov. IEEE J. Quant. Electron., 53 (6), 2400808 (2017).

[8] А.В. Бабичев, Л.Я. Карачинский, И.И. Новиков, А.Г. Гладышев, С.А. Блохин, S. Mikhailov, V. Iakovlev, A. Sirbu, G. Stepniak, L. Chorchos, J.P. Turkiewicz, K.O. Воропаев, A.C. Ионов, M. Agustin, N.N. Ledentsov, А.Ю. Егоров. Письма ЖТФ, 44 (1), 59 (2018).

[9] P. Westbergh, J.S. Gustavsson, B. Kogel, A. Hanglund, A. Larsson. IEEE J. Select. Top. Quant. Electron., 17 (6), 1603 (2011).

[10] С.А. Блохин, Н.А. Малеев, М.А. Бобров, А.Г. Кузьменков, А.В. Сахаров, В.М. Устинов. Письма ЖТФ, $44(1), 7$ (2018).

[11] С.А. Блохин, М.А. Бобров, А.А. Блохин, А.Г. Кузьменков, Н.А. Малеев, В.М. Устинов, Е.С. Колодезный, С.С. Рочас, А.В. Бабичев, И.И. Новиков, А.Г. Гладышев, Л.Я. Карачинский, Д.В. Денисов, К.О. Воропаев, А.С. Ионов, А.Ю. Егоров. Опт. и спектр., 127 (1), 145 (2019).

[12] L.A. Coldren, S.W. Corzine. Diode Lasers and Photonic Integrated Circuits (N.Y., Wiley, 1995).

[13] D. Ellafi, V. Iakovlev, A. Sirbu, S. Grigore, Z. Mickovic, A. Caliman, A. Mereuta, E. Kapon. IEEE J. Select. Top. Quant. Electron., 21 (6), 1700509 (2015).

[14] S. Spiga, D. Schoke, A. Andrejew, G. Boehm, M.-C. Amann. IEEE J. Lightwave Technol., 35 (15), 3130 (2017). 
[15] A. Grabmaier, G. Fuchs, A. Hangleiter, R.W. Glew, P.D. Greene, J.E.A. Whiteaway. J. Appl. Phys., 70 (4), 2467 (1991).

[16] M. Willatzen, T. Takahashi, Y. Arakawa. IEEE Photon. Technol. Lett., 4 (7), 682 (1992).

[17] A. Grabmaier, A. Hangleiter, G. Fuchs, J.E.A. Whiteaway, R.W. Glew. Appl. Phys. Lett., 59 (23), 3024 (1991).

[18] A. Hangleiter, A. Grabmaier, G. Fuchs. IEEE Trans. Electron Dev., 40 (11), 2106 (1993).

[19] C.Y. Tsai, Y.H. Lo, R.M. Spencer. Appl. Phys. Lett., 67 (21), 3084 (1995).

Редактор Г.А. Оганесян

\section{Influence of output optical losses on dynamic charactereistics of $1.55 \mu \mathrm{m}$ wafer-fused vertical-cavity surface-emitting lasers}

S.A. Blokhin ${ }^{1}$, M.A. Bobrov ${ }^{1}$, A.A. Blokhin ${ }^{2,1}$, A.G. Kuzmenkov ${ }^{2}$, N.A. Maleev ${ }^{1}$, V.M. Ustinov ${ }^{2}$, E.S. Kolodeznyi ${ }^{3}$, S.S. Rochas ${ }^{3}$, A.V. Babichev ${ }^{3}$, I.I. Novikov ${ }^{3}$, A.G. Gladyshev ${ }^{3}$, L.Ya. Karachinskii ${ }^{1,4}$, D.V. Denisov ${ }^{4,5}$, K.O. Voropaev ${ }^{6,7}$, A.S. Ionov ${ }^{7}$, A.Yu. Egorov ${ }^{3}$

${ }^{1}$ loffe Institute, 194021 St. Petersburg, Russia

${ }^{2}$ Research and Engineering Center for Submicron Heterostructures for Microelectronics, 194021 St. Petersburg, Russia

${ }^{3}$ ITMO University,

197101 St. Petersburg, Russia

${ }^{4}$ Connector Optics LLC,

194292 St.Petersburg, Russia

${ }^{5}$ Saint Petersburg Electrotechnical University „LETI“, 197367 St. Petersburg, Russia

${ }^{6}$ Yaroslav-the-Wise Novgorod State University, 173003 Veliky Novgorod, Russia

${ }^{7}$ OAO OKB-Planeta, 173004 Veliky Novgorod, Russia

Abstract The results of the study of the dynamic characteristics of $1.55 \mu \mathrm{m}$ single-mode vertically cavity surface emitting lasers (VCSELs), which were made by wafer fusion of high-quality Bragg reflectors based of $\mathrm{GaAs} / \mathrm{AlGaAs}$ and the active region based on thin highly strained InGaAs/InAlGaAs quantum wells, are presented. It has been found that the proposed design of the active region and the optical microcavity fundamentally make it possible to achieve a high level of differential laser gain in the temperature range of $20-85^{\circ} \mathrm{C}$, but weak electron localization leads to an increase in the gain compression at high temperatures. As a result, at $20^{\circ} \mathrm{C}$, the VCSEL modulation bandwidth can be increased from $9.2 \mathrm{GHz}$ to $11.5 \mathrm{GHz}$ by increasing output optical losses, while at $85^{\circ} \mathrm{C}$, the VCSEL modulation bandwidth does not exceed $8.5 \mathrm{GHz}$, weakly depends on the output optical losses and is mainly limited by the saturation of the optical gain. 